\title{
COMPARISON BETWEEN TWO METHODS OF CIRCUMCISION- CONVENTIONAL (DORSAL SLIT AND EXCISION) VS. GUILLOTINE
}

\author{
Ashwinkumar Shankerdan Gadhvi' ${ }^{1}$ Kamlesh Jamanadas Galani², Bhargav Ashwinbhai Trivedi ${ }^{3}$
}

${ }_{1}^{1}$ Associate Professor, Department of Surgery, CUSMC and H, Surendranagar, Gujarat, India.

${ }^{2}$ Assistant Professor, Department of Surgery, CUSMC and H, Surendranagar, Gujarat, India.

${ }^{3}$ Resident, Department of Surgery, CUSMC and H, Surendranagar, Gujarat, India.

\begin{abstract}
BACKGROUND

Circumcision is the surgical removal of the prepuce (foreskin), either in whole or in part. Religion, cultural, medical and recently public health reasons are known to be the major indications of the procedure.(1,2) Circumcision is one of the commonest procedures done now-a-day by surgeons. Various methods of circumcision are dorsal slit technique, plastibell technique, hollister bell technique, Guillotine method, sleeve technique etc. Each method has its own advantage as well as disadvantage. So, we have done a comparative study between two methods of circumcision namely Conventional (Dorsal Slit Method) and Guillotine for various aspects.
\end{abstract}

\section{MATERIALS AND METHODS}

Study was non-randomised controlled trial including 131 patients. Group A underwent circumcision by Conventional method (i.e. Dorsal slit) and Group B underwent by Guillotine method. The study and the sample size were taken for convenience during the study.

\section{RESULTS}

Post-operative pain would be moderate in majority of patients with Guillotine circumcision as compared to mild pain in Conventional circumcision on post-operative day 3. On long follow-up, pain was less with Conventional method (3.04\%) compared to Guillotine method (26.15\%). Post-operative oedema was less with Conventional method (1.52\%) compared to Guillotine method (26.15\%). Cosmetic appearance was better with Conventional method (72.21\%) compared to Guillotine method (44.61\%) on long follow-up.

\section{CONCLUSION}

Conventional method has a statistically and clinically less pain, less post-operative complication and better cosmetic outcome compared to Guillotine method on one-month follow-up. So, Conventional (i.e. Dorsal slit) method should be preferred over Guillotine method for circumcision.

\section{KEY WORDS}

Circumcision, Conventional (i.e. Dorsal Slit) Method, Guillotine Method, Methods of Circumcision. HOW TO CITE THIS ARTICLE: Gadhvi AS, Galani KJ, Trivedi BA. Comparison between two methods of circumcision- conventional (dorsal slit and excision) vs. guillotine. J. Evolution Med. Dent. Sci. 2018;7(31):3448-3451, DOI: 10.14260/jemds/2018/777

\section{BACKGROUND}

Circumcision (from Latin "circumcidere" meaning to cut around) is one of the most ancient and common surgical procedures worldwide. Circumcision is practiced since ancient times for various indications whether religious or therapeutic. It can be done with various methods by using various materials. Various methods of circumcision are dorsal slit technique, plastibell technique, Hollister bell technique, Guillotine method, Sleeve technique etc. Each method has its own advantage as well as disadvantage. Each method has its own complication. Here, I have studied two methods of circumcision namely Conventional (Dorsal Slit Method) and Guillotine and compared the results for various aspects.

'Financial or Other Competing Interest': None.

Submission 20-06-2018, Peer Review 14-07-2018,

Acceptance 20-07-2018, Published 28-07-2018.

Corresponding Author:

Kamlesh Jamanadas Galani,

\#16, Madhav Park,

Karmanpara, New Junction Road,

Surendranagar-363001,

Gujarat, India.

E-mail: drkamleshpatel8@gmail.com

DOI: $10.14260 /$ jemds/2018/777

\section{MATERIALS AND METHODS}

In the present non-randomised controlled trial, protocol of trial procedure was formed along with proforma, patient information sheet and informed consent. Total of 131 cases were taken for study (which was the available patients for study during [01-01-2016 to 31-08-2017]) and nonrandomly divided into Group A- Conventional method and Group B- Guillotine method by inclusion and exclusion criteria. The sample size was taken for convenience during the study.

Inclusion Criteria- All cases for elective circumcision. Patients selected were of any age.

\section{Exclusion Criteria}

1. Traumatic injury to prepusal skin.

2. Inseparable adhesions between glans and prepusal skin.

3. Secondary phimosis.

4. Patients with meatal stenosis.

5. Patients with hypospadias or epispadias.

6. Patients with local skin disorders.

7. Patients with suspected malignancy.

8. Paraphimosis.

9. Emergency operations were excluded. 
Post-operative evaluation was done in terms of pain, oedema, haematoma and cosmetic appearance.

Pain Results were compared and rated according to Numeric Pain Rating Scale (NPRS) ${ }^{[3,4,5]}$ as-

Mild: Easily bearable, do not require analgesic [Score- 0 to 4] Moderate: Require oral analgesic occasionally [Score- 5 to 7] Severe: Require analgesic regularly [Score- 8 to 10]

\section{Haemostatic Result were compared and Rated as-}

Good: Absolute and immediate haemostasis.

Average: Intermediate and early haemostasis.

Poor: Unsatisfactory and late haemostasis with persistent oozing.

Cosmetic results were compared and rated by Patient and Observer Scar Assessment Scale (POSAS) ${ }^{[6]}$ as-

Good: Linear scar with minimal or no puckering

Average: Linear scar with puckering of the surrounding skin without depression.

Poor: Severe puckering and depressed irregular scar.

\section{Oedema Results were Compared and Rated as-}

Present or Absent

- Follow-up of the patients were done on $3^{\text {rd }}$ PostOperative Day (POD) and weekly for one month.

- $\quad$ Statistical analysis was done wherever applicable using standard tests of significance. Here, p-value was taken by Chi-square test.

\section{RESULTS}

Patients were divided according to age group in both groups and studied as below-

\begin{tabular}{|c|c|c|}
\hline \multirow{2}{*}{ Age Group } & \multicolumn{2}{|c|}{ Numbers of Patient } \\
\cline { 2 - 3 } & Group A & Group B \\
\hline$<10$ & 9 & 8 \\
\hline $11-20$ & 18 & 17 \\
\hline $21-30$ & 13 & 14 \\
\hline $31-40$ & 12 & 12 \\
\hline $41-50$ & 5 & 6 \\
\hline$>50$ & 8 & 8 \\
\hline \multicolumn{2}{|c|}{ Table 1. Distribution According to Age } \\
\hline
\end{tabular}

Table 1 suggests that patients were divided nonrandomly according to different age group and studied for post-operative pain, post-operative oedema, cosmetic appearance and post-operative haemostasis.

\section{Post-Operative Pain}

\begin{tabular}{|c|c|c|}
\hline Pain on POD 3 & Conventional & Guillotine \\
\hline Mild & $57.57 \%$ & $27.69 \%$ \\
\hline Moderate & $39.3 \%$ & $47.69 \%$ \\
\hline Severe & $3.03 \%$ & $24.61 \%$ \\
\hline \multicolumn{2}{|c|}{ Table 2(A). Pain on Post-Operative Day 3 }
\end{tabular}

In Table $2 \mathrm{~A}$ among 66 patients who underwent Conventional circumcision, 38 patients $(57.57 \%)$ developed mild pain, 26 patients $(39.3 \%)$ developed moderate pain, while only 2 patients $(3.03 \%)$ developed severe pain. Among 65 patients who underwent Guillotine circumcision from which 18 (27.69\%) developed mild pain, 31 (47.69\%) developed moderate pain and 16 (24.61\%) developed severe pain on post-operative day 3 .

\begin{tabular}{|c|c|c|}
\hline Pain on POD 7 & Conventional & Guillotine \\
\hline No pain & $57.57 \%$ & $24.61 \%$ \\
\hline Mild & $39.39 \%$ & $50.76 \%$ \\
\hline Moderate & $3.03 \%$ & $24.61 \%$ \\
\hline Severe & 0 & 0 \\
\hline \multicolumn{2}{|c|}{ Table 2(B). Pain on Post-Operative Day 7 }
\end{tabular}

In Table 2(B) among 66 patients who underwent Conventional circumcision, 26 patients (39.39\%) developed mild pain, 02 patients $(3.03 \%)$ developed moderate pain, while no patient developed severe pain on 7 th post-operative day. Moreover 38 (57.57) patients were totally pain free on post-operative day 7. While among 65 patients who underwent Guillotine circumcision from which 33 (50.76\%) developed mild pain, 16 (24.61\%) developed moderate pain and no patient had severe pain on post-operative day 7 . Moreover, 16 patients (24.61\%) were completely pain free.

\begin{tabular}{|c|c|c|}
\hline Pain after 1 Month & Conventional & Guillotine \\
\hline No pain & $96.96 \%$ & $73.84 \%$ \\
\hline Mild & $3.03 \%$ & $26.15 \%$ \\
\hline Moderate & 0 & 0 \\
\hline Severe & 0 & 0 \\
\hline \multicolumn{2}{|c|}{ Table 2(C). Pain after 1 Month } \\
\hline
\end{tabular}

In Table 2(C) among 66 patients who underwent Conventional circumcision, $64(96.96 \%)$ patients had no pain at all. Only 2 patients (3.03\%) developed mild pain, while none had even moderate or severe pain. While among 65 patients who underwent Guillotine circumcision, 48 patients (73.84\%) had no pain at all and 17 patients $(26.15 \%)$ had mild pain, while no one was having moderate or severe pain.

\section{Post-Operative Oedema}

\begin{tabular}{|c|c|c|}
\hline Local Oedema on POD3 & Conventional & Guillotine \\
\hline Present & $66.15 \%$ & $90.76 \%$ \\
\hline Absent & $34.84 \%$ & $9.23 \%$ \\
\hline \multicolumn{2}{|c|}{ Table 3(A). Oedema on Post-Operative Day 3 } \\
\hline
\end{tabular}

In Table 3(A) among 66 patients who underwent Conventional circumcision, 43 (66.15\%) developed local operative site oedema, while 23 (34.84\%) did not develop it. Among 65 patients who underwent Guillotine circumcision $59(90.76 \%)$ developed local oedema, while only $6(9.23 \%)$ does not develop it.

\begin{tabular}{|c|c|c|}
\hline Local Oedema on POD 7 & Conventional & Guillotine \\
\hline Present & $24.24 \%$ & $75.38 \%$ \\
\hline Absent & $75.75 \%$ & $24.61 \%$ \\
\hline \multicolumn{2}{|c|}{ Table 3(B). Oedema on Post-Operative Day 7 } \\
\hline
\end{tabular}

In Table 3(B) among 66 patients of Conventional circumcision $16(24.24 \%)$ was having oedema, while 50 $(75.75 \%)$ was having no oedema. Among 65 patients of Guillotine circumcision 49 (75.38\%) were having oedema, while only 16 patients (24.61\%) were not having oedema.

\begin{tabular}{|c|c|c|}
\hline $\begin{array}{c}\text { Local Oedema after 1 } \\
\text { Month }\end{array}$ & Conventional & Guillotine \\
\hline Present & $1.51 \%$ & $26.15 \%$ \\
\hline Absent & $98.48 \%$ & $73.84 \%$ \\
\hline \multicolumn{2}{|c|}{ Table 3(C). Oedema after 1 Month } \\
\hline
\end{tabular}


In Table 3 (C) from 66 patients of Conventional method, only $1(1.51 \%)$ patient had persistence local oedema after 1 month of surgery, while $65(98.48 \%)$ were free of oedema. Conversely, from 65 of Guillotine 17 patients (26.15\%) were having persistence oedema, while 48 (73.84\%) was oedema free.

\section{Cosmetic Appearance (CA)}

\begin{tabular}{|c|c|c|}
\hline CA after POD 3 & Conventional & Guillotine \\
\hline Good & $53.03 \%$ & $23.07 \%$ \\
\hline Average & $45.45 \%$ & $56.92 \%$ \\
\hline Poor & $1.51 \%$ & $20 \%$ \\
\hline Table 4(A). Cosmetic appearance on Post-Operative Day 3 \\
\hline
\end{tabular}

In Table 4(A) among 66 patients who underwent Conventional circumcision, 35 (53.03\%) had good cosmetic appearance, $30(45.45 \%)$ had average and 1 (1.51\%) had poor cosmetic appearance. While among 65 patients who underwent Guillotine circumcision, 15 (23.07\%) had good cosmetic appearance, 37 (56.92\%) had average appearance and $13(20 \%)$ had poor cosmetic appearance.

\begin{tabular}{|c|c|c|}
\hline CA on POD 7 & Conventional & Guillotine \\
\hline Good & $62.12 \%$ & $32.30 \%$ \\
\hline Average & $37.87 \%$ & $49.23 \%$ \\
\hline Poor & 0 & $18.46 \%$ \\
\hline Table 4(B). Cosmetic appearance on Post-Operative Day 7 \\
\hline
\end{tabular}

In Table 4(B), among 66 patients who underwent Conventional circumcision 41 (62.12\%) had good cosmetic appearance, 25 (37.87\%) had average and none had poor cosmetic appearance. While among 65 patients who underwent Guillotine circumcision, 21 (32.30\%) had good cosmetic appearance, 32 (49.23\%) had average appearance and $12(18.46 \%)$ had poor cosmetic appearance.

\begin{tabular}{|c|c|c|}
\hline CA after 1 Month & Conventional & Guillotine \\
\hline Good & $72.21 \%$ & $38.46 \%$ \\
\hline Average & $28.78 \%$ & $44.61 \%$ \\
\hline Poor & 0 & $16.92 \%$ \\
\hline Table 4(C). Cosmetic appearance after 1 Month \\
\hline
\end{tabular}

In Table 4(C), among 66 patients who underwent Conventional circumcision 47 (72.21\%) had good cosmetic appearance, $19(28.78 \%)$ had average and none had poor cosmetic appearance. While among 65 patients who underwent Guillotine circumcision, 25 (38.46\%) had good cosmetic appearance, 29 (44.61\%) had average appearance and $11(16.92 \%)$ had poor cosmetic appearance.

\section{Post-Operative Haemostasis}

\begin{tabular}{|c|c|c|}
\hline Haemostasis on POD 3 & Conventional & Guillotine \\
\hline Good & $77.27 \%$ & $38.46 \%$ \\
\hline Average & $21.21 \%$ & $40 \%$ \\
\hline Poor & $1.51 \%$ & $21.53 \%$ \\
\hline \multicolumn{2}{|c|}{ Table 5(A). Haemostasis on Post-Operative Day 3 } \\
\hline
\end{tabular}

In Table 5(A) from 66 patients who underwent Conventional circumcision, 51 (77.27\%) was having good haemostasis, $14(21.21 \%)$ having average haemostasis and only $1(1.51 \%)$ had poor haemostasis. While from 65 patients who underwent Guillotine circumcision, 25 (38.46\%) were having good haemostasis, $26(40 \%)$ were having average haemostasis, while $14(21.53 \%)$ were having poor haemostasis.

\begin{tabular}{|c|c|c|}
\hline Haemostasis on POD 7 & Conventional & Guillotine \\
\hline Good & $93.93 \%$ & $73.84 \%$ \\
\hline Average & $6.06 \%$ & $24.61 \%$ \\
\hline Poor & 0 & $1.55 \%$ \\
\hline Table 5(B). Haemostasis on Post-Operative Day 7 \\
\hline
\end{tabular}

In Table 5(B) among 66 patients who underwent Conventional circumcision, 62 (93.93\%) had good haemostasis and only $4(6.06 \%)$ were having average haemostasis, while none was having poor haemostasis. Among 65 patients underwent Guillotine circumcision, 48 (73.84\%) were having good haemostasis, 16 (24.61\%) were having average haemostasis and 1 (1.55\%) had poor haemostasis on post-operative day 7 .

\section{DISCUSSION}

Our study of comparison of two methods of circumcision was conducted at the Department of Surgery at tertiary care hospital of a medical college after getting approval from Scientific Review Committee and Ethical Committee (Human Research) of the Institute.

- It was conducted from $1 / 1 / 2016$ to $31 / 08 / 2017$.

- 131 patients undergoing planned surgery were included in the study according to inclusion criteria.

Our Observation and Results were analysed and it is discussed here-

Post-operative pain would be moderate in majority of patients with Guillotine circumcision as compared to mild in Conventional circumcision on post-operative day 3 ( $\mathrm{p}$ value0.000173). However, majority of patients with Conventional circumcision are pain free on post-operative day 7 , while only few i.e. $24.61 \%$ patients are complete pain free on postoperative day 7 (p value- 0.000098). After 1 month, almost all i.e. $96.96 \%$ patients were pain free in Conventional circumcision, while $73.84 \%$ patients were pain free and $26.15 \%$ still have mild pain ( $\mathrm{p}$ value- 0.000171 ).

Post-operative local oedema is almost inevitable in Guillotine circumcision as $90.76 \%$ would be having it on postoperative day 3 compared to $66.15 \%$ incidence in Conventional circumcision ( $\mathrm{P}$ value- 0.000414). It almost completely resolves after 1 month in Conventional method, i.e. $98.48 \%$ patients would have no oedema after 1 month, compared to $26.15 \%$ of Guillotine circumcision would be still having it after 1 month (P value- 0.000042 ).

Cosmetic appearance are compared after 1 month where all signs of inflammation subside completely and $72.21 \%$ of Conventional group has good cosmetic appearance, while rest i.e. $28.78 \%$ having average appearance and none was having poor cosmetic appearance compared to good cosmetic appearance only in $38.46 \%$. While majority were i.e. $44.61 \%$ having average cosmetic appearance with $16.92 \%$ having poor cosmetic appearance in Guillotine group ( $\mathrm{P}$ value0.05576).

Post-operative haemostasis is far better in Conventional method, as $77.27 \%$ achieve immediate good haemostasis on post-operative day 3 compared to only $38.46 \%$ of Guillotine method circumcision ( $P$ value- 0.00001). Bleeding is more 
common with the excision method and when less attention is placed on haemostasis during the procedure. $[7,8]$

\section{CONCLUSION}

- Conventional method of circumcision is causing less pain compared to Guillotine method.

- Conventional method has less haemorrhagic complications than Guillotine method.

- Conventional method has fewer incidences of postoperative oedema compared to Guillotine method and also oedema resolved faster in Conventional method.

- Post-operative appearance is far better in Conventional method having linear scar and proper egg-shell appearance in majority of cases compared to average scar in Guillotine method.

Circumcision has low complication rates when performed properly, $1 \%$ minor complication rate when performed in children and less than $0.5 \%$ minor complication when performed in infancy. ${ }^{[9]}$ So, Conventional, i.e. dorsal slit and excision method is preferable to Guillotine method for circumcision.

\section{REFERENCES}

[1] Soliman AH. A comparative study of the female genital system of Mammalia [MD Thesis]. Cairo, Egypt: Department of Anatomy, Cairo University 1956.
[2] Martin RD. Primate origins and evolution: a phylogenetic reconstruction. Princeton, New Jersey: Princeton University Press 1990: p. 42-3.

[3] Childs JD, Piva SR, Fritz JM. Responsiveness of the numeric pain rating scale in patients with low back pain. Spine (Phila Pa 1976) 2005;30(11):1331-4.

[4] Jensen MP, McFarland CA. Increasing the reliability and validity of pain intensity measurement in chronic pain patients. Pain 1993;55(2):195-203.

[5] Rodriguez CS. Pain measurement in the elderly: a review. Pain Manag Nurs 2001;2(2):38-46.

[6] Draaijers LJ, Tempelman FR, Botman YA, et al. The patient and observer scar assessment scale: a reliable and feasible tool for scar evaluation. Plast Reconstr Surg 2004;113(7):1960-5.

[7] Weiss H, Polonsky J, Bailey R, et al. Male circumcision: global trends and determinants of prevalence, safety and acceptability. World Health Organization and the Joint United Nations Programme on HIV/AIDS (UNAIDS) 2007.

[8] Manual for male circumcision under local anaesthesia. Geneva: World Health Organization, 2008. [Last accessed 2012 Dec 01]. WHO/UNAIDS/JHPIEGO.

[9] Weiss HA, Larke N, Halperin D, et al. Complications of circumcision in male neonates, infants and children: a systematic review. BMC Urol 2010;10:2. 